\title{
Disfagia orofaríngea em pacientes submetidos à entubação orotraqueal
}

\section{Oropharyngeal dysphagia in patients submitted to orotracheal intubation}

\author{
Michele Ramos Grigio Kunigk ${ }^{1}$, Ethel Chehter $^{2}$
}

\begin{abstract}
RESUMO
Objetivo: Detectar e caracterizar as alterações da fase oral e faríngea da deglutição, bem como verificar a ocorrência de penetração e aspiração laríngeas em pacientes submetidos à entubação orotraqueal. Métodos: O estudo incluiu 30 adultos internados na unidade de tratamento intensivo do Hospital Estadual Mário Covas, que receberam entubação orotraqueal no período de 40 horas a 15 dias. Todos foram submetidos, por duas vezes, à avaliação endoscópica da deglutição, na primeira e segunda semanas, após a extubação, sendo observadas as alterações da fase oral e faríngea da deglutição e a presença de penetração e aspiração laríngeas. Resultados: Na primeira avaliação, as alterações da fase oral estiveram presentes em 19(63,3\%) pacientes e, na segunda, em 12(40\%). As alterações da fase faríngea ocorreram na primeira avaliação em $27(90 \%)$ pacientes e, na segunda, em 8(26,7\%). A penetração e a aspiração laríngeas foram detectadas nas duas avaliações, sendo diferentes para cada consistência e volume testados. Conclusão: A população submetida à entubação orotraqueal após a extubação apresenta alterações das fases oral e faríngea da deglutição caracterizadas por uma variedade de comprometimentos e acompanhadas de penetração e aspiração laríngeas.
\end{abstract}

DESCRITORES: Transtornos da deglutição/etiologia; Transtornos da deglutição/ diagnóstico; Respiração artificial/efeitos adversos; Respiradores mecânicos/utilização

\section{INTRODUÇÃO}

A entubação orotraqueal é comumente utilizada nas unidades de tratamento intensivo em pacientes graves que necessitam de auxílio para a manutenção da respiração; porém, apesar dos benefícios, pode causar ao trato respiratório superior complicações significantes para determinar prejuízos no processo da deglutição ${ }^{(1-2)}$.

O processo da deglutição envolve estruturas ósseas, musculares e cartilaginosas do trato digestório e respiratório, atuando de forma coordenada para encaminhar o bolo alimentar até o estômago, sendo que qualquer desequilíbrio pode determinar prejuízos à sua função ${ }^{(3-5)}$.

A entubação orotraqueal prolongada pode proporcionar lesões na cavidade oral, faringe e laringe, que causam diminuição da motricidade e da sensibilidade local e comprometem o processo da deglutição, determinando as disfagias orofaríngeas. Estas podem desencadear problemas como a desnutrição e a pneumonia aspirativa, piorando significantemente o estado clínico do paciente internado.

Trabalho realizado na Faculdade de Medicina do ABC - FMABC - Santo André (SP), Brasil.

(1) Mestre, Fonoaudióloga do Setor de Disfagia Neonatal, Pediátrica e Adulto do Hospital Estadual Mário Covas - Santo André (SP), Brasil.

(2) Professor Adjunto da Faculdade de Medicina do ABC - FMABC - Santo André (SP), Brasil.

Recebido em: 6/11/2006; Aceito: 10/10/2007
A introdução da alimentação por via oral, nos pacientes que fizeram uso de tubo orotraqueal, deve ser cuidadosa a fim de garantir a nutrição adequada e evitar complicações respiratórias. Dessa forma, realizamos esse estudo com o objetivo de detectar e caracterizar as alterações da fase faríngea da deglutição, bem como verificar a ocorrência de penetração e aspiração laríngeas em pacientes submetidos à entubação orotraqueal.

\section{MÉTODOS}

Para a realização deste estudo, o projeto foi enviado ao Comitê de Ética da Faculdade de Medicina do ABC, o qual foi aprovado sob o registro 202/2005.

Neste estudo prospectivo, aberto e seqüencial, foram incluídos 30 pacientes, internados na Unidade de Tratamento Intensivo do Hospital Estadual Mário Covas de Santo André, submetidos à entubação orotraqueal por mais de 48 horas e até 15 dias, já que, após esse tempo, é necessária a realização da traqueostomia.

Foram considerados como critérios de inclusão: adultos com tempo de extubação entre dois e sete dias, estabilizados clinicamente e liberados pela equipe médica para avaliação da alimentação por via oral. Foram excluídos do estudo os pacientes portadores de doenças degenerativas, traqueostomia, dificuldades de alimentação prévia à internação e que tivessem recebido alimentação por via oral após a extubação. 
Todos os pacientes foram transportados pela equipe da unidade de internação até o Setor de Otorrinolaringologia do próprio Hospital e acomodados em cadeira própria para o exame otorrinolaringológico, sendo que, na impossibilidade da retirada do paciente da maca, este foi posicionado sobre ela em decúbito elevado a $90^{\circ}$.

Do total de adultos avaliados $20(66,6 \%)$ eram do sexo masculino e $10(33,3 \%)$ do feminino, com idade de 20 a 72 anos e média 48 anos. Foi verificada a ocorrrência de 18 (60\%) traumatismos cranioencefálicos, $4(13,3 \%)$ acidentes vasculares cerebrais do tipo isquêmico e hemorrágico, $2(6,7 \%)$ tumores cerebrais, 2 (6,7\%) paradas cardiorespiratórias, 2 (6,7\%) insuficiências coronarianas, 1 (3,3\%) lesão raquimedular e 1 $(3,3 \%)$ insuficiência respiratória aguda.

Para a realização dos exames foi utilizado um aparelho de nasofibrolaringoscopia flexível, da marca Pentax, de origem japonesa, modelo PNL 10RD3, e as imagens foram gravadas por meio de um vídeo cassete Semp Toshiba modelo X687, de origem brasileira, e uma televisão de 12 polegadas da marca LG, modelo Cinemaster.

Durante os exames, foram oferecidos alimentos de consistência pastosa, semi-pastosa, liquida e sólida com corante alimentar e preparados a partir da diluição em água de suco em pó. Para se chegar à consistência pastosa, foram adicionadas três colheres de espessante de alimentos em $40 \mathrm{ml}$ do suco preparado; para a consistência semi-pastosa, foram adicionadas duas colheres de espessante para o mesmo volume de suco. Para avaliação da consistência sólida, foi utilizada bolacha sem recheio.

Os alimentos de consistências amolecidas, assim como os líquidos, foram oferecidos na colher, visto que, durante o exame, não foi possível beber no copo devido ao posicionamento do aparelho. A bolacha foi oferecida em pedaços, diretamente na cavidade oral do paciente.

Os adultos incluídos neste estudo foram submetidos em dois momentos ao exame de videoendoscopia da deglutição, que foi realizado pelo médico otorrinolaringologista e acompanhado por duas fonoaudiólogas. As duas avaliações foram realizadas, respectivamente, na primeira e segunda semanas, após a extubação.

Para a realização da videoendoscopia da deglutição, o fibroscópio foi introduzido sem anestésico tópico, através da narina do paciente e posicionado de forma a visualizar as regiões de hipofaringe e laringe. A seguir, o avaliador ofereceu os alimentos com a colher, seguindo a ordem de avaliação de consistências: pastoso, semi-pastoso, líquido e sólido, nos volumes de 5 e $10 \mathrm{ml}$. Na ocorrência de penetração laríngea sem limpeza efetiva e espontânea de via aérea, ou aspiração traqueal, o exame era interrompido, pois o paciente apresentava riscos para alimentação.

A avaliação da fase oral da deglutição, realizada no momento do exame, foi feita a partir da observação da movimentação e do posicionamento das estruturas orofaciais durante o processo de deglutição.

A avaliação da fase faríngea da deglutição foi realizada, posteriormente, a partir da análise da gravação da videoendoscopia da deglutição até se chegar a um consenso dos três profissionais presentes durante o exame.
Foram observadas as seguintes alterações:

- escape oral anterior: ausência ou diminuição de vedamento labial antes ou durante a deglutição que ocasione escape de alimento para fora da cavidade oral;

- redução do controle motor oral: incapacidade ou lentidão dos movimentos da língua e da mandíbula durante a preparação e posicionamento do bolo em cavidade oral antes de ser deglutido;

- estase em cavidade oral: presença de alimentos em cavidade oral, após a deglutição;

- redução da sensibilidade oral: ausência ou diminuição de resposta de limpeza dos lábios após o toque do alimento nos lábios, ou ausência da resposta de limpeza da cavidade oral após a deglutição (estases alimentares);

- escape posterior: escape de alimentos da cavidade oral para a faringe, antes do início da fase faríngea da deglutição;

- atraso no início da fase faríngea: presença da ejeção do bolo alimentar, antes do fechamento laríngeo;

- estase em parede posterior da faringe: presença de resíduos alimentares na região da parede posterior da faringe, após três deglutições;

- redução de sensibilidade laringo-faríngea: ausência de resposta de deglutição, tosse ou náusea ao toque do fibroscópio em epiglote, pregas ariepiglóticas e aritenóides;

- estase em recessos faríngeanos: presença de resíduos alimentares em valéculas e seios piriformes, após três deglutições;

- penetração laríngea: presença de alimento desde a face laríngea da epiglote até o limite das pregas vocais;

- aspiração laríngea: passagem do alimento abaixo das pregas vocais;

A análise estatística dos dados foi realizada pelo teste qui-quadrado, sendo considerados estatisticamente significantes os valores d" 0,05 .

\section{RESULTADOS}

A média do tempo de uso de sonda gástrica (oral ou nasal) até a data do primeiro exame foi de 16,4 dias e da entubação orotraqueal, de 11,2 dias, sendo que $8(26,7 \%)$ pacientes foram extubados acidentalmente e, em seguida, reentubados.

O intervalo de tempo entre a primeira e segunda avaliação foi, em média, de sete dias. Na primeira avaliação, o tempo médio de extubação foi de 4,1 dias e, na segunda, 10,9 dias, havendo diferença estatisticamente significante $(\mathrm{p}=0,00)$.

$\mathrm{Na}$ primeira avaliação, as alterações da fase oral da deglutição estiveram presentes em 19 (63,3\%) pacientes e, na segunda, em 12(40\%), conforme caracterizado na Tabela 1.

Conforme a Tabela 1, as alterações da fase faríngea da deglutição estiveram presentes na primeira avaliação, em 27 (90\%) pacientes e, na segunda, em 8 (26,7\%).

A penetração e a aspiração laríngeas ocorreram nas duas avaliações, conforme apresentado nas Tabelas 2 e 3, e esti- 
Tabela 1. Presença das alterações nas fases oral e faríngea da deglutição na primeira e segunda avaliações

\begin{tabular}{|c|c|c|c|c|c|}
\hline \multirow[t]{2}{*}{ Alterações } & \multicolumn{2}{|c|}{ Primeira avaliação } & \multicolumn{2}{|c|}{ Segunda avaliação } & \multirow[t]{2}{*}{$\mathrm{p}$} \\
\hline & $\mathrm{N}$ & $\%$ & $\mathrm{~N}$ & $\%$ & \\
\hline Escape oral anterior & 15 & $50,0 \%$ & 6 & $20,0 \%$ & 0,16 \\
\hline Redução do controle motor oral & 18 & $60,0 \%$ & 11 & $36,7 \%$ & 0,73 \\
\hline Estase em cavidade oral & 17 & $56,7 \%$ & 5 & $16,7 \%$ & 0,01 \\
\hline Redução da sensibilidade oral & 17 & $56,7 \%$ & 4 & $13,3 \%$ & 0 \\
\hline Escape posterior & 18 & $60,0 \%$ & 6 & $20,0 \%$ & 0,02 \\
\hline Atraso no início da fase faríngea & 16 & $53,3 \%$ & 5 & $16,7 \%$ & 0,03 \\
\hline Estase em parede posterior de faringe & 10 & $33,3 \%$ & 0 & $0,0 \%$ & 0,001 \\
\hline Estase em recessos faringeanos & 22 & $73,3 \%$ & 3 & $10,0 \%$ & 0 \\
\hline Redução da sensibilidade laringo-faríngea & 26 & $86,7 \%$ & 4 & $13,3 \%$ & 0 \\
\hline
\end{tabular}

$\mathrm{N}=$ número de pacientes; Teste qui-quadrado $\mathrm{p}<0,05$

veram mais presentes para a consistência líquida, totalizando $6(46,2 \%)$ pacientes na primeira avaliação $(5 \mathrm{ml})$ e $4(14,8 \%)$ na segunda $(10 \mathrm{ml})$.

Tabela 2. Presença de penetração laríngea na primeira e segunda avaliações.

\begin{tabular}{lcccc}
\hline $\begin{array}{l}\text { Consistências e } \\
\text { Volumes }\end{array}$ & \multicolumn{2}{c}{$\begin{array}{c}\text { Primeira Avaliação } \\
\text { Presente }\end{array}$} & \multicolumn{2}{c}{$\begin{array}{c}\text { Segunda Avaliação } \\
\text { Presente }\end{array}$} \\
& $\mathrm{N}$ & $\%$ & $\mathrm{~N}$ & $\%$ \\
\hline Pastoso $5 \mathrm{~mL}$ & 7 & 23,3 & 1 & 3,3 \\
Pastoso $10 \mathrm{~mL}$ & 3 & 12,5 & 0 & 0,0 \\
Semi pastoso $5 \mathrm{~mL}^{*}$ & 8 & 36,4 & 1 & 3,4 \\
Semi pastoso $10 \mathrm{~mL}^{*}$ & 4 & 26,7 & 0 & 0,0 \\
Líquido $5 \mathrm{~mL}^{*}$ & 7 & 53,8 & 3 & 10,7 \\
Líquido $10 \mathrm{~mL}^{*}$ & 4 & 50,0 & 8 & 30,8 \\
Sólido $1 / 4{ }^{*}$ & 0 & 0,0 & 0 & 0,0 \\
Sólido $1 / 2$ * & 0 & 0,0 & 0 & 0,0 \\
\hline
\end{tabular}

$\mathrm{N}=$ número de pacientes; Teste qui-quadrado: $\mathrm{p}<0,05$

Tabela 3. Presença de aspiração laríngea na primeira e segunda avaliações

\begin{tabular}{lcccc}
\hline $\begin{array}{l}\text { Consistências e } \\
\text { Volumes }\end{array}$ & \multicolumn{2}{c}{$\begin{array}{c}\text { Primeira Avaliação } \\
\text { Presente }\end{array}$} & \multicolumn{2}{c}{$\begin{array}{c}\text { Segunda Avaliação } \\
\text { Presente }\end{array}$} \\
& $\mathrm{N}$ & $\%$ & $\mathrm{~N}$ & $\%$ \\
\hline Pastoso $5 \mathrm{~mL}^{*}$ & 7 & 23,3 & 1 & 3,3 \\
Pastoso $10 \mathrm{~mL}^{*}$ & 2 & 8,3 & 0 & 0,0 \\
Semi pastoso $5 \mathrm{~mL}^{*}$ & 7 & 31,8 & 1 & 3,7 \\
Semi pastoso $10 \mathrm{~mL}^{*}$ & 1 & 6,3 & 0 & 0,0 \\
Líquido $5 \mathrm{~mL}^{*}$ & 6 & 46,2 & 2 & 7,1 \\
Líquido $10 \mathrm{~mL}^{*}$ & 3 & 37,5 & 4 & 14,8 \\
Sólido $1 / 4{ }^{*}$ & 0 & 0,0 & 0 & 0,0 \\
Sólido $1 / 2{ }^{*}$ & 0 & 0,0 & 0 & 0,0 \\
\hline
\end{tabular}

$\mathrm{N}=$ número de pacientes; Teste qui-quadrado: $\mathrm{p}<0,05$

\section{DISCUSSÃO}

Este estudo mostrou que pacientes submetidos à entubação orotraqueal apresentam alterações na fase oral e faríngea da deglutição, freqüentemente acompanhadas de penetração e aspiração laríngeas, sendo comum a presença de mais de uma alteração para cada adulto avaliado, determinando falta de funcionalidade no processo da deglutição. Estudos realizados com pacientes extubados têm resultados semelhantes e mostram que a disfagia orofaríngea presente nesta população não é caracterizada por um tipo específico de distúrbio, mas por uma variedade de comprometimentos orofaríngeos $^{(6-9)}$.

A fase oral da deglutição é responsável pela contenção, preparo e posicionamento do bolo alimentar, e a fase faríngea é responsável pelo seu transporte até o esôfago e pelo fechamento das vias aéreas, protegendo-as contra a penetração e aspiração laríngeas ${ }^{(10-11)}$, sendo que as alterações nestas fases foram encontradas em grande parte da população incluída nesse estudo. Essas alterações podem ser decorrentes da presença do tubo endotraqueal, que pode determinar lesões, causando a diminuição da motricidade e da sensibilidade da cavidade oral, laringe e faringe. Além disso, as funções relacionadas a essas estruturas, como a respiração, a fala e a deglutição, não são realizadas durante a instituição da entubação orotraqueal, propiciando diminuição da função laríngea e conseqüente inatividade da musculatura no período de sua utilização ${ }^{(12)}$.

Os resultados mostraram que, na primeira e segunda avaliações, a redução do controle motor oral foi a alteração mais encontrada na fase oral da deglutição. Essa função é realizada pelos órgãos envolvidos na fase oral, principalmente a língua, que realiza movimentos misturando o bolo alimentar com a saliva e posicionando-o em seu centro para que ocorra a deglutição. Estudo ${ }^{(7)}$ realizado mostrou que pacientes submetidos à entubação orotraqueal com e sem alteração neurológica apresentaram, como maior comprometimento da fase oral, a redução do controle da língua, sendo o resultado semelhante ao deste estudo.

Apesar da diminuição de todas as alterações da fase oral da primeira para a segunda avaliação, a redução da sensibilidade oral foi a mais significante. O maior tempo após a extubação e a retomada das funções relacionadas à cavidade oral como a fala, respiração e a deglutição, podem ter auxiliado na melhora desta alteração.

A redução da sensibilidade laringo-faríngea foi altamente constatada na fase faríngea da deglutição, na primeira avaliação e pode estar relacionada com a interrupção da passa- 
gem do ar por essa estrutura, já que, durante a instituição da ventilação mecânica invasiva, a passagem do mesmo é realizada pelo tubo endotraqueal. Além disso, estudos nasofibrolaringoscópicos mostram que anormalidades, como o edema e a ulceração da região laríngea, são extremamente comuns nesses pacientes e que essas alterações podem ser suficientes para comprometer a sensibilidade laringo-faríngea e prejudicar o mecanismo de proteção das vias aéreas no processo da deglutição ${ }^{(6-9)}$.

A alteração da fase faríngea que mais apresentou redução de ocorrência entre as avaliações foi a redução da sensibilidade laringo-faríngea. Esse fato pode estar relacionado com o tempo de extubação, já que seu aumento pode melhorar as complicações advindas da entubação orotraqueal. Além disso, a retomada das funções do trato respiratório superior pode também contribuir para a melhora da sensibilidade laringo-faríngea ${ }^{(9)}$.

As penetrações e aspirações laríngeas foram freqüentemente encontradas e ocorreram mais para a consistência líquida. Na segunda avaliação, foi observada a redução desses episódios, sendo que a melhora do controle motor e da sensibilidade orofaríngea verificada neste estudo pode ter sido determinante para esta redução da entrada dos alimentos nas vias aéreas. Estudos mostram que a penetração e a aspiração laríngeas ocorrem pela dimuição ou incoordenação do fechamento laríngeo, durante o processo da deglutição e são fatores de risco para pneumonias, podendo levar o paciente a comprometimentos pulmonares letais ${ }^{(13-21)}$.

Os resultados revelaram que as alterações da deglutição, bem como a penetração e aspiração laríngeas, foram menos freqüentes após a segunda semana de extubação, podendo estar relacionadas à melhora do controle motor e da sensibilidade oral após a retirada da entubação orotraqueal. Estudos sugerem que as alterações da deglutição, em pacientes submetidos à ventilação invasiva, são passageiras de acordo com o aumento do tempo de extubação, porém esse tempo ainda não está determinado ${ }^{(9)}$.
Apesar das limitações dessa pesquisa, devido à heterogeneidade da amostra em relação à idade, doença de base e co-morbidades, alterações da deglutição acompanhadas de penetrações e aspirações laríngeas foram encontradas nessa população, caracterizando a presença de disfagia orofaríngea. Assim, a introdução de alimentos por via oral, nas primeiras semanas após a extubação, é de risco para complicações nutricionais e respiratórias devido à falta de funcionalidade da deglutição.

A liberação da dieta ao paciente e a retirada da sonda de alimentação devem ser realizadas por profissional especializado, que irá avaliar a funcionalidade das estruturas orofaríngeas e do processo da deglutição, a fim de garantir sua funcionalidade e segurança.

Será que esse cuidado com a alimentação após a extubação poderia diminuir os agravantes à saúde do paciente, o seu tempo e internação, o uso de medicamentos, bem como diminuir os gastos hospitalares?

Estudos controlados devem ser realizados com a finalidade de criar um protocolo de alimentação aos pacientes que foram submetidos à entubação orotraqueal, com o objetivo de determinar a consistência alimentar e o momento mais seguro para o início da alimentação por via oral.

\section{CONCLUSÃO}

O estudo concluiu que a população submetida à ventilação mecânica invasiva após a extubação apresenta alterações das fases oral e faríngea da deglutição caracterizadas por uma variedade de comprometimentos e acompanhadas por penetração e aspiração laríngeas.

\section{AGRADECIMENTOS}

Agradecemos à Dra. Priscila Bogar Rapoport, Dra.Zelita Caldeira Ferreira Guedes e Dr. Ronaldo Frizzarini pela contribuição científica.

\begin{abstract}
Purpose: To detect and characterize alterations in the oropharyngeal phase of swallowing as well as to verify the occurrence of laryngeal penetration and aspiration in patients submitted to invasive mechanical ventilation. Methods: The study consisted of 30 individuals hospitalized in the intensive care unit of the Mario Covas State Hospital, who received invasive mechanical ventilation. They were all submitted twice to endoscopic evaluation for swallowing disorders in the first and second week after extubation. Alterations were observed in the oro-pharyngeal phase of swallowing and also the presence of penetration and laryngeal aspiration. Results: In the first evaluation, alterations in the oral phase were observed in 19 patients (63.3\%) and in the second evaluation, in $12(40 \%)$ patients. Alterations in the pharyngeal phase were present in the first evaluation in $27(90 \%)$ and in the second evaluation, in $8(26.7 \%)$ patients. The penetration and laryngeal aspiration were present in both evaluations and resulted different for each tested meal consistency and volume. Conclusion: The population submitted to invasive mechanical ventilation after extubation presents alterations in the oropharyngeal phase of swallowing characterized by several impairments and followed by penetration and laryngeal aspiration.
\end{abstract}

KEYWORDS: Deglutition disorders/etiology; Deglutition disorders/diagnosis; Respiration, artificial/adverse effects; Ventilators, mechanical/utilization 


\section{REFERÊNCIAS}

1. Pompílio CE, Carvalho CRR. Ventilação mecânica: definição e classificação. In: Carvalho CRR, editor. Ventilação mecânica. São Paulo: Atheneu; 2000. p. 125-33.

2. Zin WA, Rocco PRM. Histórico, indicações, tendências atuais. In: Auler Júnior JOC, Amaral RVG. Assistência ventilatória mecânica. São Paulo: Atheneu; 1998. p. 66-102.

3. Dantas RO. Disfagia orofaríngea. In: Macedo Filho ED, Pissani JC, Carneiro HJ, Gomes FG. Disfagia: abordagem multidisciplinar. 2a ed. São Paulo: Frontis Editorial; 1999. p.19-29.

4. Smithard DG, O’Neill PA, Parks C, Morris J. Complications and outcome after acute stroke. Does dysphagia matter? Stroke. 1996;27(7):1200-4. Erratum in: Stroke. 1998;29(7):1480-1.

5. Yamada EK, Siqueira KO, Xerez D, Koch HA, Costa MMB. A influência das fases oral e faríngea na dinâmica da deglutição. Arq Gastroenterol. 2004;41(1):18-23.

6. Leder SB, Cohn SM, Moller BA. Fiberoptic endoscopic documentation of the high incidence of aspiration following extubation in critically ill trauma patients. Dysphagia. 1998;13(4):208-12.

7. Tolep K, Getch CL, Criner GJ. Swallowing dysfunction in patients receiving prolonged mechanical ventilation. Chest. 1996;109(1):16772.

8. Partik B, Pokieser P, Schima W, Schoeber E, Stadler A, Eisenhuber E, et al. Videofluoroscopy of swallowing in symptomatic patients who have undergone long-term intubation. AJR Am $\mathrm{J}$ Roentgenol. 2000;174(5):1409-12.

9. DeVita MA, Spierer-Rundback L. Swallowing disorders in patients with prolonged orotracheal intubation or tracheostomy tubes. Crit Care Med. 1990;18(12):1328-30

10. Junqueira P. A importância da fase oral na dinâmica da deglutição. In: Costa MMB, Castro LP. Tópicos em deglutição e disfagia. Rio de Janeiro: Medsi; 2003. p.31-6.

11. Medda BK, Kern M, Ren J, Xie P, Ulualp SO, Lang IM, Shaker R. Relative contribution of various airway protective mechanisms to prevention of aspiration during swallowing. Am J Physiol Gastrointest Liver Physiol. 2003;284(6):G933-9.
12. Colice GL, Stukel TA, Dain B. Laryngeal complications of prolonged intubation. Chest. 1989;96(4):877-84. Comment in: Chest. 1990;98(3):776-7.

13. Horner J, Massey EW, Riski JE, Lathrop DL, Chase KN. Aspiration following stroke: clinical correlates and outcome. Neurology. 1988;38(9):1359-62.

14. Pikus L, Levine MS, Yang YX, Rubesin SE, Katzka DA, Laufer I, Gefter WB. Videofluoroscopic studies of swallowing dysfunction and the relative risk of pneumonia. AJR Am J Roentgenol. 2003;180(6):16136. Comment in: AJR Am J Roentgenol. 2004;182(1):257; author reply 257.

15. Power ML, Hamdy S, Singh S, Tyrrell PJ, Turnbull I, Thompson DG. Deglutitive laryngeal closure in stroke patients. J Neurol Neurosurg Psychiatry. 2007;78(2):141-6.

16. Jafari S, Prince RA, Kim DY, Paydarfar D. Sensory regulation of swallowing and airway protection: a role for the internal superior laryngeal nerve in humans. J Physiol. 2003;550(Pt 1):287-304.

17. Myssiorek D. Recurrent laryngeal nerve paralysis: anatomy and etiology. Otolaryngol Clin North Am. 2004;37(1):25-44.

18. Sulica L. The superior laryngeal nerve: function and dysfunction. Otolaryngol Clin North Am. 2004;37(1):183-201.

19. Elpern EH, Scott MG, Petro L, Ries MH. Pulmonary aspiration in mechanically ventilated patients with tracheostomies. Chest. 1994;105(2):536-6.

20. Martin-Harris B, McMahon SJ, Haynes R. Aspiration and dysphagia: pathophysiology and outcome. Phonoscope. 1998;1:123-32.

21. Mari F, Matei M, Ceravolo MG, Pisani A, Montesi A, Provinciali L. Predictive value of clinical indices in detecting aspiration in patients with neurological disorders. J Neurol Neurosurg Psychiatry. 1997;63(4):456-60. 